\title{
D-Dimer-Test: Effizienz gesteigert
}

\author{
Durch eine geschickte Teststrategie \\ unter Berücksichtigung der kli- \\ nischen Prätest-Wahrscheinlichkeit \\ gelingt es, die Effizienz des D-Dimer- \\ Tests in der Diagnostik der tiefen \\ Beinvenenthrombose erheblich zu \\ steigern.
}

- Die Bestimmung von Fibrin-Spaltprodukten mithilfe des D-Dimer-Tests ist ein sensitives, aber wenig spezifisches Verfahren für die Diagnose der tiefen Beinvenenthrombose (TVT). Ein negatives Testergebnis (D-Dimer $<0,5 \mu \mathrm{g} /$ ml) schließt diese Diagnose aus, ein positives Testergebnis erfordert jedoch ein weiteres diagnostisches Verfahren, in der Regel die Kompressionssonografie.

In einer randomisierten und kontrollierten Studie an 1720 ambulanten und stationären Patienten fünf kanadischer Krankenhäuser mit Verdacht auf erstmals aufgetretene TVT wurde die Strategie einer allgemeinen Testung mittels D-Dimer-Test verglichen mit dem Vorgehen auf der Basis einer Prätest-Wahrscheinlichkeit und einer Anhebung des Cut-off des D-Dimer-Tests bei ambulanten Patienten.

Bei 860 Patienten wurde die Strategie des einheitlichen D-Dimer-Tests verfolgt. In dieser Gruppe betrachtete man die TVT bei einem D-Dimer von $<0,5$ $\mu \mathrm{g} / \mathrm{ml}$ als ausgeschlossen, und es wurde keine Antikoagulation durchgeführt. Ein Wert über dieser Schwelle führte zur Kompressionssonografie der proximalen Venen am symptomatischen Bein, die bei initial negativem Ergebnis und mäßiger bis hoher Wahrscheinlichkeit 6-8 Tage später wiederholt wurde.

In der gleich starken Studiengruppe hob man bei ambulanten Patienten mit geringer Wahrscheinlichkeit den Cut-off des D-Dimer-Tests auf $1,0 \mu \mathrm{g} / \mathrm{ml}$ an. Bei ambulanten Patienten mit hoher Wahrscheinlichkeit für eine TVT und bei allen stationären Patienten unterließ man

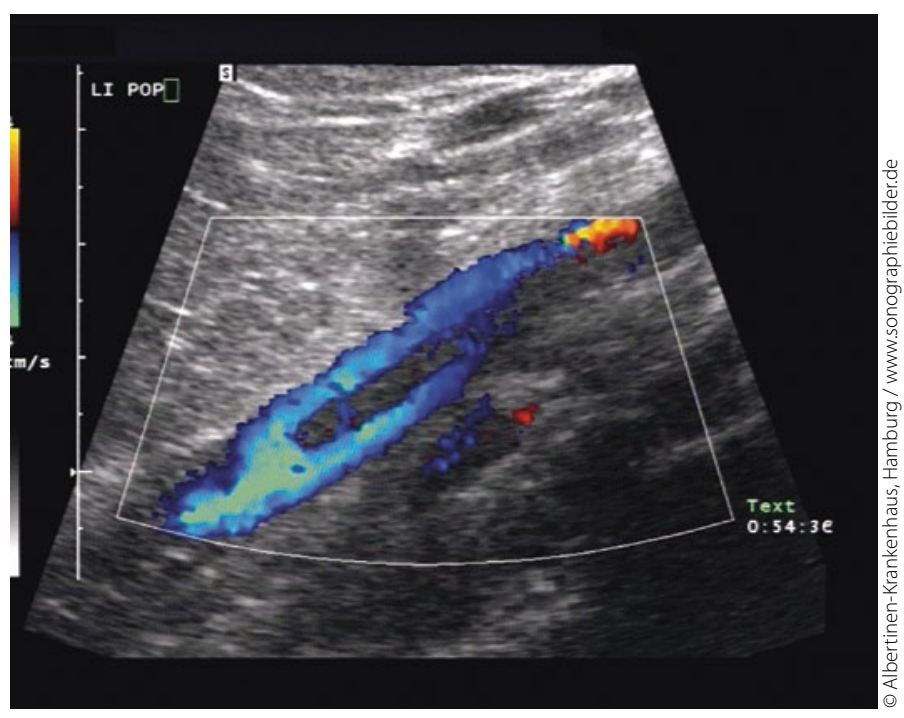

Ist die Wahrscheinlichkeit hoch, dass eine tiefe Beinvenenthrombose vorligt, sollte man besser gleich sonografieren.

dagegen den D-Dimer-Test und führte direkt eine Kompressionssonografie durch. Ambulante Patienten mit mäBiger Wahrscheinlichkeit für eine TVT wurden nach dem gängigen Cut-off von $0,5 \mu \mathrm{g} / \mathrm{ml}$ klassifiziert.

In beiden Studiengruppen betrug die Inzidenz der symptomatischen TVT $0,5 \%$. Mit dem selektiven Vorgehen konnten 21,8\% der D-Dimer-Tests und $7,6 \%$ der Ultraschalluntersuchungen eingespart werden. Bei den ambulanten Patienten mit niedriger Wahrscheinlich-

\section{Kommentar}

Der anfangs mit großer Begeisterung propagierte Einsatz des D-Dimer-Tests in der Diagnostik der TVT ist mittlerweile einer gewissen Ernüchterung gewichen. Einmal mehr mussten wir die Erfahrung machen, dass es in der Medizin nur ganz selten vorkommt, dass man eine Diagnose mit einem einzigen diagnostischen Verfahren bestätigen oder ausschließen kann. Vor Durchführung des D-Dimer-Tests sollte man auf alle Fälle den Patienten anhand des Wells-Score bezüglich der Wahrscheinlichkeit für eine TVT keit konnten sogar 21\% der Kompressionssonografien vermieden werden. Als praktische Konsequenz aus der Studie ergibt sich, dass bei ambulanten Patienten mit geringer Wahrscheinlichkeit auf eine erste TVT der Cut-off des DDimer auf $1,0 \mu \mathrm{g} / \mathrm{ml}$ angehoben werden sollte. Bei ambulanten Patienten mit hoher Prätest-Wahrscheinlichkeit und bei allen stationären Patienten kann man sich den D-Dimer-Test sparen und sollte den Patienten direkt sonografisch untersuchen.

klassifizieren. Bei hoher Wahrscheinlichkeit kann man sich den D-Dimer-Test sparen, bei geringer Wahrscheinlichkeit sollte zur Vermeidung zu vieler falsch positiver Ergebnisse der Cut-off angehoben werden.

H. S. FÜESSL

- L.-A. Linkins et al.

(Korres.: linkinla@mcmaster.ca): Selective DDimer Testing for diagnosis of a first suspected episode of deep venous thrombosis. Ann Intern Med. 2013; 158: 93-100. 